\title{
On uniformly convex functions
}

\author{
by A. W. Goodman (Tampa, Fla.)
}

\begin{abstract}
We introduce a new class of normalized functions regular and univalent in the unit disk. These functions, called uniformly convex functions, are defined by a purely geometric property. We obtain a few theorems about this new class and we point out a number of open problems.
\end{abstract}

1. Introduction. An earlier paper [3] introduced the class UST of uniformly starlike functions. We now consider the similar concept of uniformly convex functions. Let CV denote the usual class of convex functions

$$
f(z)=z+\sum_{n=2}^{\infty} a_{n} z^{n}
$$

These are normalized functions regular and univalent in $E:|z|<1$, for which $f(E)$ is a convex domain.

Definition 1. A function $f(z)$ is said to be uniformly convex in $E$ if $f(z)$ is in CV and has the property that for every circular arc $\gamma$ contained in $E$, with center $\zeta$ also in $E$, the arc $f(\gamma)$ is a convex arc. We let UCV denote the class of all such functions.

A directed arc $\Gamma(t), a<t<b$, is said to be convex if the argument of the tangent to $\Gamma(t)$ is a nondecreasing function of $t$ [2, Vol. I, pp. 109-110]. In our case the direction of $\Gamma(t)=f(\gamma)$ is the one dictated by the direction of $\gamma$ which is the usual counterclockwise direction on a circle.

In $[2$, p. 110$]$ we proved that if any arc $\gamma$ is given by $z(t)$, then $f(\gamma)$ is convex iff

$$
\operatorname{Im}\left[\frac{z^{\prime \prime}(t)}{z^{\prime}(t)}+\frac{f^{\prime \prime}(z)}{f^{\prime}(z)} z^{\prime}(t)\right] \geq 0
$$

for all $z$ on $\gamma$.

1991 Mathematics Subject Classification: Primary 30C45; Secondary 30C50.

Key words and phrases: univalent functions, convex functions, coefficient bounds. 
For a circular arc with center $\zeta$, set $z=\zeta+r e^{i t}$. Then $z^{\prime}(t)=i(z-\zeta)$ and $z^{\prime \prime}(t)=-(z-\zeta)$. A brief computation using (1.2) will give

THEOREM 1. Let $f(z)$ have the form (1.1). Then $f(z)$ is in UCV iff

$$
1+\operatorname{Re}\left[\frac{f^{\prime \prime}(z)}{f^{\prime}(z)}(z-\zeta)\right] \geq 0
$$

for every pair $(z, \zeta)$ in the polydisc $E \times E$.

Thus all the properties of functions in UCV are contained implicitly in the relation (1.3). However, obtaining these properties is not always easy.

2. Functions with positive real part on the polydisc. Let $P^{(2)}$ denote the set of functions

$$
P(z, \zeta)=1+\sum_{m+n>0} \sum_{m n} z^{m} \zeta^{n}
$$

that are regular in $E \times E$ and satisfy the condition $\operatorname{Re} P \geq 0$ in that domain. Such functions have been the subject of numerous investigations. However, a representation formula for all functions in $P^{(2)}$ is still missing [4].

As my colleague V. Totik suggested, if we set $\zeta=e^{i \alpha} z$ in (2.1) we obtain

$$
F(z) \equiv P\left(z, e^{i \alpha} z\right) \equiv 1+\sum_{n=1}^{\infty} B_{n} z^{n}
$$

where for $n \geq 1$

$$
B_{n}=\sum_{k=0}^{n} b_{n-k, k} e^{i k \alpha} .
$$

If $P(z, \zeta) \in P^{(2)}$, the classical Carathéodory Theorem applied to $F(z)$ gives $\left|B_{n}\right| \leq 2$ for all $n \geq 1$, and all real $\alpha$. Then on integrating $B_{n} \bar{B}_{n}$ on a suitable circle we obtain

$$
\sum_{k=0}^{n}\left|b_{n-k, k}\right|^{2} \leq 4, \quad n \geq 1,
$$

for $P(z, \zeta)$ in $P^{(2)}$.

Let

$$
Q(z, \zeta) \equiv 1+\frac{f^{\prime \prime}(z)}{f^{\prime}(z)}(z-\zeta)
$$

and let $Q^{(2)}=\{Q(z, \zeta) \mid f(z) \in \mathrm{UCV}\}$. Then $Q^{(2)}$ is properly contained in $P^{(2)}$ and in fact if $Q(z, \zeta)$ has the form $(2.1)$, then clearly $b_{m n}=0$ for all $m \geq 0$ and $n \geq 2$. Further, if we put $\zeta=z$ we see that $b_{m-1,1}=-b_{m, 0}$ for all $m \geq 1$. Although these restrictions on $Q(z, \zeta)$ may be interesting, apparently they are not very helpful in obtaining properties for the class UCV. 
3. Properties of uniformly convex functions. It is clear from the definition of UCV that the class is invariant under the rotation $e^{i \alpha} f\left(e^{-i \alpha} z\right)$. Hence we may assume w.l.o.g. that $a_{2} \geq 0$ in (1.1). We can let $\zeta \rightarrow 1^{-}$ in (2.5) and obtain

$$
\operatorname{Re}(Q(z, 1))=\operatorname{Re}\left(1-2 a_{2}+\sum_{n=1}^{\infty} c_{n} z^{n}\right) \geq 0 .
$$

This gives $0 \leq 1-2 a_{2}$ or $0 \leq a_{2} \leq \frac{1}{2}$. But if $a_{2}=\frac{1}{2}$, then $c_{n}=0$ for all $n>0$ and hence

$$
1+z \frac{f^{\prime \prime}(z)}{f^{\prime}(z)}(z-1) \equiv 0
$$

Now (3.2) holds if and only if $f(z)=-\ln (1-z)$. For this function $Q(z, \zeta)=$ $(1-\zeta) /(1-z)$ and for suitable selection of $z$ and $\zeta$ we have $\operatorname{Re} Q(z, \zeta)<0$. We have proved

Theorem 2. If $f(z) \in \mathrm{UCV}$, then $\left|a_{2}\right|<1 / 2$.

The sharp upper bounds for $\left|a_{n}\right|$ in the class UCV are not known, but we have

THEOREM 3. If $f(z)$ is in UCV, then $\left|a_{n}\right| \leq 1 / n$ for every $n \geq 2$.

Pr o of. We use the symbol $f(z) \ll g(z)$ to indicate that the power series for $f(z)$ is dominated by the power series for $g(z)$ [2, Vol. I, pp. 81-83]. We set $\zeta=-z$ in $Q(z, \zeta)$ and if $f(z) \in \mathrm{UCV}$, then

$$
Q(z, \zeta)=Q(z,-z)=1+\frac{f^{\prime \prime}(z)}{f^{\prime}(z)} 2 z \ll \frac{1+z}{1-z} .
$$

Then

$$
2 z \frac{f^{\prime \prime}(z)}{f^{\prime}(z)} \ll \frac{2 z}{1-z} .
$$

Integration gives $\ln f^{\prime}(z) \ll-\ln (1-z)$. Consequently $f^{\prime}(z) \ll 1 /(1-z)$ and hence $\left|a_{n}\right| \leq 1 / n$.

The following example will be useful.

THEOREM 4. The function

$$
F_{1}(z)=\frac{z}{1-A z}=z+\sum_{n=2}^{\infty} A^{n-1} z^{n}
$$

is in $\mathrm{UCV}$ iff $|A| \leq 1 / 3$.

Proof. By a rotation we may assume that $0 \leq A$ in (3.5). A simple computation shows that for this function

$$
Q(z, \zeta)=\frac{1+A z-2 A \zeta}{1-A z}
$$


We set $z=r e^{i \theta}$ and $\zeta=\rho e^{i \varphi}$. Then $\operatorname{Re} Q(z, \zeta) \geq 0$ iff

$$
\operatorname{Re}\left(1+A r e^{i \theta}-2 A \rho e^{i \varphi}\right)\left(1-A r e^{-i \theta}\right) \geq 0
$$

or

$$
1-2 A \rho \cos \varphi-A^{2} r^{2}+2 A^{2} r \rho \cos (\varphi-\theta) \geq 0 .
$$

It is clear that the minimum of the expression on the left side of (3.6) occurs when $r=\rho=1, \varphi=0$ and $\theta=\pi$. (Thus, $\zeta=1$ and $z=-1$.) These values yield $1-2 A-3 A^{2} \geq 0$, and this is true for $0 \leq A \leq 1 / 3$. Thus, the condition is sufficient for $F_{1}(z)$ to be in UCV. By a limit argument as $z \rightarrow-1^{+}$and $\zeta \rightarrow 1^{-}$the condition is also necessary.

As a corollary of Theorem 4 we see that the set UCV has infinitely many members.

It is natural to look for transformations which preserve the set UCV. The rotation $e^{i \alpha} f\left(e^{-i \alpha} z\right)$ is one such, but no other transformation seems to be available. Pommerenke [2, Vol. II, p. 109] introduced the concept of the linear-invariant family $M$ and showed that numerous theorems about the family $M$ followed immediately once we have proved that $M$ is a linearinvariant family. By definition $M$ is a linear-invariant family if

$$
\Lambda_{\varphi}[f] \equiv \frac{f(\varphi(z))-f(\varphi(0))}{\varphi^{\prime}(0) f^{\prime}(\varphi(0))}, \quad \varphi(z)=\frac{z+c}{1+\bar{c} z},
$$

is also in $M$ for every $f$ in $M$ and every $c$ in $E$. If we apply (3.7) to $F_{1}(z)=z /(1-A z)$ we find that

$$
\Lambda_{\varphi}\left[\frac{z}{1-A z}\right]=\frac{z}{1-B z}, \quad B=\frac{A-\bar{c}}{1-c A} .
$$

Now set $A=1 / 4<1 / 3$ and $c=-1 / 2$. Then $F_{1}(z)$ is in UCV, but $B=$ $2 / 3>1 / 3$, so $\Lambda_{\varphi}$ is not in UCV. We have proved

THEOREM 5. The family UCV is not a linear-invariant family.

4. The sets UST and UCV. We recall the classic Alexander Theorem that if $f(z)$ is given by (1.1), then $f(z)$ is in CV iff

$$
F(z) \equiv z f^{\prime}(z)
$$

is in ST, where CV and ST are the usual normalized families of convex and starlike functions. What is the situation when we prefix the word "uniformly"?

To prove that (4.1) does not give a one-to-one correspondence between the sets UST and UCV we need two examples. 
LEMMA 1. The function $F(z) \equiv z-B z^{2}$ is in UST iff $|B| \leq \sqrt{3} / 4$.

As we mentioned in [3], the proof of this lemma is a simple exercise in calculus, but the computation is rather long so we omit the details.

Lemma 2. The function $f(z) \equiv z-A z^{2}$ is in UCV iff $|A| \leq 1 / 6$.

Proof. If $f(z)=z-A z^{2}$, then

$$
1+\operatorname{Re}\left[\frac{f^{\prime \prime}(z)}{f^{\prime}(z)}(z-\zeta)\right]=1+\operatorname{Re} \frac{-2 A(z-\zeta)}{1-2 A z} .
$$

But for $0<A<1 / 2$,

$$
1+\operatorname{Re} \frac{-2 A(z-\zeta)}{1-2 A z} \geq 1-\left|\frac{2 A(z-\zeta)}{1-2 A z}\right| \geq 1-\frac{4 A}{1-2 A}
$$

and hence in $\bar{E} \times \bar{E}$

$$
1+\operatorname{Re} \frac{f^{\prime \prime}(z)}{f^{\prime}(z)}(z-\zeta) \geq \frac{1-6 A}{1-2 A} \geq 0
$$

iff $A \leq 1 / 6$. But equality can occur when $z=1$ and $\zeta=-1$.

Now set $F(z)=z-B z^{2}$ where $B=\sqrt{3} / 4$ and hence $F(z)$ is in UST. Then (4.1) gives $f(z)=z-A z^{2}$ where $A=\sqrt{3} / 8 \approx 0.216$. Since $\sqrt{3} / 8>1 / 6$, the corresponding $f(z)$ is not in UCV. The converse relation may hold. It may be that $f(z)$ in UCV implies that $F(z)=z f^{\prime}(z)$ is in UST, but I have not been able to prove or disprove this statement.

5. A sufficient condition. It is well-known [1] that if $f(z)$ is given by (1.1) and $\sum_{n=2}^{\infty} n\left|a_{n}\right| \leq 1$, then $f(z)$ is in ST. In [3] we proved that if

$$
\sum_{n=2}^{\infty} n\left|a_{n}\right| \leq \frac{\sqrt{2}}{2}
$$

then $f(z)$ is in UST. However, it was conjectured in [3] that the constant $\sqrt{2} / 2$ can be replaced by $\sqrt{3} / 2$ in $(5.1)$.

TheOREM 6. Suppose that $f(z)$ is given by (1.1). If

$$
\sum_{n=2}^{\infty} n(n-1)\left|a_{n}\right| \leq \frac{1}{3}
$$

then $f(z)$ is in UCV. Further, the constant $1 / 3$ in (5.2) cannot be replaced by a larger number. 
Proof. If (5.2) is satisfied, then $\sum_{n=2}^{\infty} n\left|a_{n}\right| \leq 1 / 3$. Hence in $\bar{E} \times \bar{E}$

$$
\begin{aligned}
1+\operatorname{Re} \frac{f^{\prime \prime}(z)}{f^{\prime}(z)}(z-\zeta) \geq 1-\frac{\sum_{n=2}^{\infty} n(n-1)\left|a_{n}\right|\left|z^{n-2}\right|}{1-\sum_{n=2}^{\infty} n\left|a_{n}\right|\left|z^{n-1}\right|}|z-\zeta| \\
\geq 1-\frac{2 / 3}{1-1 / 3}=0 .
\end{aligned}
$$

But equality is attained in (5.3) when $f(z)=z-z^{2} / 6, z=1$ and $\zeta=-1$.

\section{References}

[1] A. W. Goodman, Univalent functions and nonanalytic curves, Proc. Amer. Math. Soc. 8 (1957), 598-601.

[2] - Univalent Functions, Polygonal Publ. Co. Inc., Washington, N.J., 1983.

[3] -, On uniformly starlike functions, J. Math. Anal. Appl. 155 (1991), 364-370.

[4] W. Rudin, Function Theory in Polydiscs, Benjamin, New York 1969.

DEPARTMENT OF MATHEMATICS

UNIVERSITY OF SOUTH FLORIDA

TAMPA, FLORIDA 33620-5700

U.S.A. 